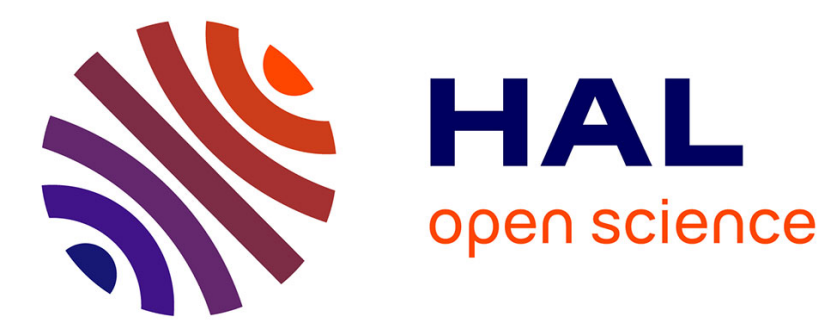

\title{
Compact and accurate concept of laser wavemeters based on ellipsometry
}

Karim Ouedraogo, Suat Topsu, Luc Chassagne, Pascal Ruaux, Barthélemy

Cagneau, Yasser Alayli

\section{- To cite this version:}

Karim Ouedraogo, Suat Topsu, Luc Chassagne, Pascal Ruaux, Barthélemy Cagneau, et al.. Compact and accurate concept of laser wavemeters based on ellipsometry. Review of Scientific Instruments, 2011, 82, pp.055102. 10.1063/1.3585975 . hal-00830879

\section{HAL Id: hal-00830879 \\ https://hal.science/hal-00830879}

Submitted on 5 Jun 2013

HAL is a multi-disciplinary open access archive for the deposit and dissemination of scientific research documents, whether they are published or not. The documents may come from teaching and research institutions in France or abroad, or from public or private research centers.
L'archive ouverte pluridisciplinaire HAL, est destinée au dépôt et à la diffusion de documents scientifiques de niveau recherche, publiés ou non, émanant des établissements d'enseignement et de recherche français ou étrangers, des laboratoires publics ou privés. 


\title{
Compact and accurate concept of laser wavemeters based on ellipsometry
}

\author{
K. Ouedraogo, S. Topsu, L. Chassagne, P. Ruaux, B. Cagneau, and Y. Alayli \\ LISV/University of Versailles, 78035 Versailles, France
}

(Received 1 February 2011; accepted 31 March 2011; published online 4 May 2011)

\begin{abstract}
Common laser wavemeters are based on a scanning Michelson interferometer. Displacements of the moving mirror as long as tens of centimeters are needed to reach a relative accuracy of $1 \times 10^{-6}(1 \sigma)$ on the unknown laser wavelengths. Such a long displacement range makes the system very sensitive to mechanical vibrations and to misalignments of the laser beams. The purpose of this paper is to demonstrate a new concept of laser wavemeter based on the measurements of the ellipsometric parameters $\psi$ and $\Delta$ of the laser beams. Experimental results show that a $10^{-6}(1 \sigma)$ accuracy level could be reach with a displacement range of only $4 \mu \mathrm{m}$. Implementations of the device are described. Comparisons between our polarimetric wavemeter and a calibrated wavemeter are presented for two lasers, an extended cavity laser diode at $656 \mathrm{~nm}$ and a $532 \mathrm{~nm}$ green line Nd:YAG laser. (c) 2011 American Institute of Physics. [doi:10.1063/1.3585975]
\end{abstract}

\section{INTRODUCTION}

Numerous designs of laser wavemeter have been proposed over the years. Most of them are based on interferometric concepts. ${ }^{1-5}$ An often used type of wavemeter is based on a Michelson interferometer. ${ }^{6-8} \mathrm{~A}$ frequency stabilized stable laser with a well-characterized wavelength is used as a reference. The reference and the unknown laser sources are injected into a Michelson interferometer and their interference fringes are simultaneously measured on different detectors. The relative lengths of the interferometer arms are varied while the number of fringes is counted for both the reference and unknown sources. The number of fringes counted over a distance $d$ is $N_{r}=2 d / \lambda_{r}$, where $\lambda_{r}$ is the wavelength in air of the reference laser. For a second laser with an unknown wavelength $\lambda_{u}, N_{u}=2 d / \lambda_{u}$ fringes would be counted. The ratio of the two fringes counts is equal to the ratio of the two wavelengths. Providing that one of the wavelengths is known, the ratio of fringes gives the wavelength of the second laser using

$$
\lambda_{u}=\frac{N_{r}}{N_{u}} \lambda_{r} .
$$

Resolution of 1 part in $10^{6}$ is achieved if one million fringes are counted, which for a $632.991 \mathrm{~nm}$ wavelength laser, means that the mirror in the Michelson interferometer must be moved over about $30 \mathrm{~cm}$ or $15 \mathrm{~cm}$ if we use a push-pull configuration. ${ }^{10}$ The scanning nature of this design leads to relatively fragile instruments. The control of the measurement process and the data analysis are usually accomplished with a microprocessor device. Various types of errors can affect the measurement accuracy. ${ }^{11}$ One of them is the length drifts and imperfections of the scanning mechanism that can introduce substantial errors. The principle of such a wavemeter can be extended for the measurement of the optical spectrum of non-monochromatic sources. The spectrum is obtained by applying a Fourier transform to the detected power versus arm length difference. This method is called Fourier transform spectroscopy. ${ }^{8}$
Most of the existing wavemeters have been optimized for resolution. Wavemeters based on the measurement of the Doppler frequency shifts ${ }^{9}$ of the output beams issue from the Michelson interferometer have demonstrated a resolution of 3 part in $10^{9}$. However, there are cases where resolution is not the key parameter. In coarse dense-wavelength divisionmultiplexing communication systems, the required resolution is such that simple dispersion spectrometers are sufficient as long as the resulting wavemeter is miniature, portable, and robust. There are robust static wavemeters, most notably those based on static Fizeau interferometer. ${ }^{12-14}$ These designs use two plane reflecting surfaces with a slight deviation from exact parallelity. They contain a glass wedge with a small angular mismatch of a few arcseconds, where the front surface is partially reflecting and the back surface is fully reflecting. There are also implementations with discrete mirrors. Two copies of the input beam are superimposed with a slight relative angle, leading to an interference pattern, the period of which depends on the wavelength. Usually, the input beam first gets through some spatial filter, and a collimated beam with large diameter is sent onto the Fizeau interferometer. The shape of the interference pattern is measured with a CCD array and the data are processed using a microprocessor. In this case, the resolution is limited by the number of pixels on the detector array, as it sets a limit on the highest spatial frequency that can be detected in the interferogram. An accuracy of 1 part in $10^{6}$ has been demonstrated but only to a limited range of wavelengths compared to the Michelson-based wavemeters. ${ }^{14,15}$ The wedge spacing of a Fizeau wavelength meter is an important parameter in the conversion of fringe patterns into wavelengths of interfering light. The major disadvantages of this excellent method are the extremely high level of precision necessary in the fabrication of the optical components and the sensitivity to temperature and to mechanical vibrations. The correction of chromatic aberrations of the Fizeau interferometer is also necessary to keep a high accuracy level over the spectral bandwidth of the device. 
Ellipsometric technics have demonstrated capabilities to improve the accuracy of homodyne interferometers. ${ }^{16,17}$ In this paper, we propose a new concept of accurate and compact laser wavemeters based on the combination of a Michelson interferometer and ellipsometry. With such a device, laser wavemeter with an accuracy level of 1 part in $10^{6}$ could be reach with a displacement range of only $4 \mu \mathrm{m}$. Implementations of the device are described. Comparisons between our polarimetric wavemeter and a calibrated wavemeter are presented for two lasers, an extended cavity laser diode at 656 $\mathrm{nm}$ and a $532 \mathrm{~nm}$ green line $\mathrm{Nd}$ :YAG laser.

\section{PRINCIPLE OF OUR POLARIMETRIC WAVEMETER}

The setup of our polarimetric wavemeter is given in Fig. 1. It consists of polarizers, beamsplitters, mirrors, quarter-waveplates, and two retroreflectors mounted on a translation stage in a push-pull configuration. The reference laser and the unknown laser are superimposed before entering in the wavemeter. Both laser beams are polarized linearly and oriented at $45^{\circ}$ in order to be separated in two equal intensity laser beams by a polarizing beamsplitter. Then each beam is retroreflected by a mirror after traveling twice through a quarter-waveplate. At the output of the wavemeter, the beams are recombined, mixed up to another quarterwaveplate and sent into polarimeter. The optical axis of the quarter-waveplates are oriented at $45^{\circ}$.

A mathematical approach of the optical setup is made using the Jones matrices calculation. ${ }^{18-21}$ Let $\vec{\xi}_{L}$ be the electromagnetic field that represents the laser beam, expressed in terms of two independent and perpendicular directions, which are referred as $s$ and $p$ directions,

$$
\overrightarrow{\xi_{L}}=\left(\begin{array}{c}
\xi_{s} \\
\xi_{p}
\end{array}\right)=\left(\begin{array}{c}
\left|\xi_{o s}\right| e^{i\left(\omega t-k z+\phi_{s}\right)} \\
\left|\xi_{o p}\right| e^{i\left(\omega t-k z+\phi_{p}\right)}
\end{array}\right) .
$$

These two directions lie in a plane perpendicular to the propagation one. The direction $p$ is at $90^{\circ}$ with respect to the direction of propagation, while direction $s$ points out of the plane of the page. The polarization state of the laser beam can be characterized by two parameters $(\psi, \Delta)$ known as the ellipsometric parameters (Fig. 2). The parameters tan $(\psi)$ and

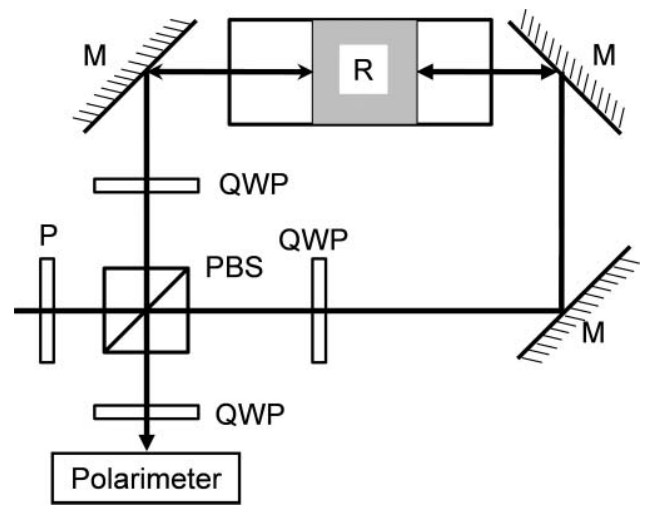

FIG. 1. Setup of the polarimetric wavemeter: $\mathbf{M}$ mirror, $\mathbf{P}$ polarizer, $\mathbf{P B S}$ polarizing beamsplitter, $\mathbf{Q W P}$ quarter-waveplate, and $\mathbf{R}$ moving retroreflectors.

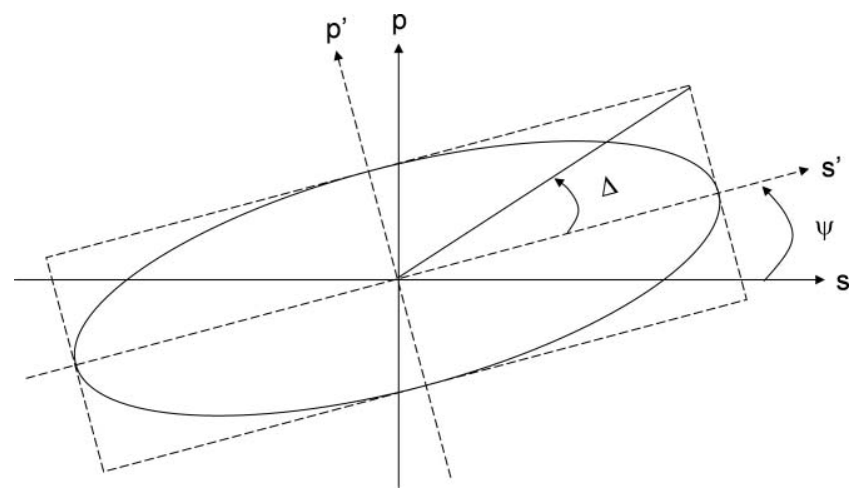

FIG. 2. Definition of the ellipsometric parameters ellipticity $\Delta$ and azimuth $\psi$.

$\Delta$ represent, respectively, the amplitude ratio and the phase difference of the $\xi_{p}$ and $\xi_{s}$ components of the electromagnetic field. There are several methods for determining these parameters. The polarimeter we used in this paper (PA450, Thorlabs), consists of a spinning quarter-waveplate and employs Fourier analysis of the incoming laser beam to calculate $\psi$ and $\Delta$ from the amplitudes and the phases of the second and fourth harmonics of the signal. ${ }^{22}$

Here we consider only ideal optical components. Physical effects that have the same influence on both polarizations, such as absorption, can in fact be taken into account by means of reference measurements. Omitting the temporal and spatial propagation terms, the Jones matrices of the two laser beams issue from the field of the reference laser $\vec{\xi}_{R}$ at the output of the wavemeter are

$$
\begin{gathered}
\vec{\xi}_{R, 1}=\frac{1}{\sqrt{2}}\left(\begin{array}{c}
\exp \left(\mp i k \delta_{R}\right) \\
-i \exp \left(\mp i k \delta_{R}\right)
\end{array}\right), \\
\vec{\xi}_{R, 2}=\frac{1}{\sqrt{2}}\left(\begin{array}{c}
\exp \left( \pm i k \delta_{R}\right) \\
i \exp \left( \pm i k \delta_{R}\right)
\end{array}\right),
\end{gathered}
$$

where $\delta_{R}$ represents the path difference covered by the two reference laser beams after the polarizing beamsplitter (Fig. 1). The total electromagnetic field of the reference laser at the output of the interferometer is

$$
\vec{\xi}_{R}=\vec{\xi}_{R, 1}+\vec{\xi}_{R, 2}=\left(\begin{array}{c}
\cos \left(k \delta_{R}\right) \\
\mp \sin \left(k \delta_{R}\right)
\end{array}\right) .
$$

Furthermore, at the output of the wavemeter, we have two components circularly polarized with a phase difference equal to $\left(\pi / 2 \pm 2 k \delta_{R}\right)$ resulting to a linear polarization state. Hence, the electromagnetic field at the output of the apparatus could also be written as

$$
\vec{\xi}_{R}=\left(\begin{array}{c}
\cos \psi_{r} \\
\sin \psi_{r}
\end{array}\right)
$$

where $\psi_{r}$ is the angle between the polarization plane and the $s$ - axis (Fig. 2). Using Eqs. (5) and (6), we can see that the resulting polarization state of the electromagnetic field is linear and will rotate with an angle $\psi_{r}$ in regard to the $s$-axis as $k \delta_{r}$ varies, i.e., as the mirror moves. 


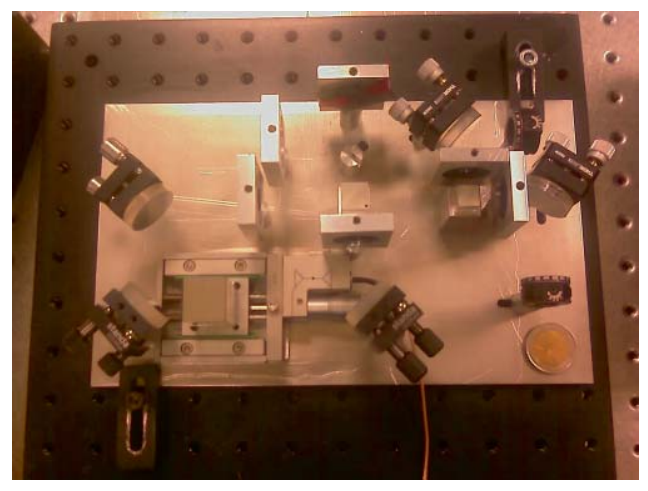

FIG. 3. (Color online) Photographic view of the polarimetric wavemeter.

The value of $\psi_{R}$ over a displacement $d$ of the moving retroreflectors is $\psi_{r}=k \times 4 d$, where $k=2 \pi / \lambda_{r}$. We suppose that this laser has been calibrated and its wavelength in vacuum $\lambda_{0 r}$ is known. For a second laser with an unknown wavelength in air $\lambda_{u}, \psi_{u}$ would be determined. The ratio of $\psi$ is equal to the ratio of the wavelengths. Hence,

$$
\lambda_{u}=\frac{\psi_{r}}{\psi_{u}} \lambda_{r} .
$$

In vacuum, it becomes

$$
\lambda_{0 u}=\frac{\psi_{r}}{\psi_{u}} \lambda_{0 r}\left(\frac{n_{u}}{n_{r}}\right),
$$

where $n_{r}$ and $n_{u}$ are the refractive index of air of respectively the reference and the unknown wavelengths.

The ellipsometric parameter $\psi_{r, u}$ can be measured with a resolution of $0.01^{\circ}$ using currently commercially available ellipsometers. Taking $\lambda_{r}=632.991 \mathrm{~nm}$, to reach a resolution of 1 part in $10^{6}$ on the unknown laser wavelength, the displacement of the moving retroreflectors has to be at least of $4.4 \mu \mathrm{m}$. As such displacements are easily achievable using piezoelectric actuators, a realization of a very robust and compact wavemeters becomes possible. Furthermore, our system becomes less sensitive to the fluctuations of the refractive index of air. ${ }^{11}$ In terms of comparison, the same resolution level with a scanning Michelson wavemeter needs a mechanical displacement of about $15 \mathrm{~cm}$ that is to say 30000 times longer.

Figure 3 represents a photography of the laboratory prototype of our polarimetric wavemeter.

\section{EXPERIMENTAL RESULTS AND DISCUSSIONS}

\section{A. Stability and resolution}

We measure the stability and the noise level of our apparatus in order to deduce its resolution. Our device is based on the use of a commercial polarimeter (TXP500, Thorlabs). The stability of our wavemeter mainly depends on the stability of the polarimeter. To determine its stability, we send into the polarimeter head a laser with a fixed polarization state. A Glan-Thompson polarizing prism with an extinction ratio of $10^{-5}$ has been used. The value of $\psi$ is then recorded from the polarimeter over an integration time of $\tau=6 \mathrm{~ms}$ for each measurement point. A number of $K=3200$ samples has been

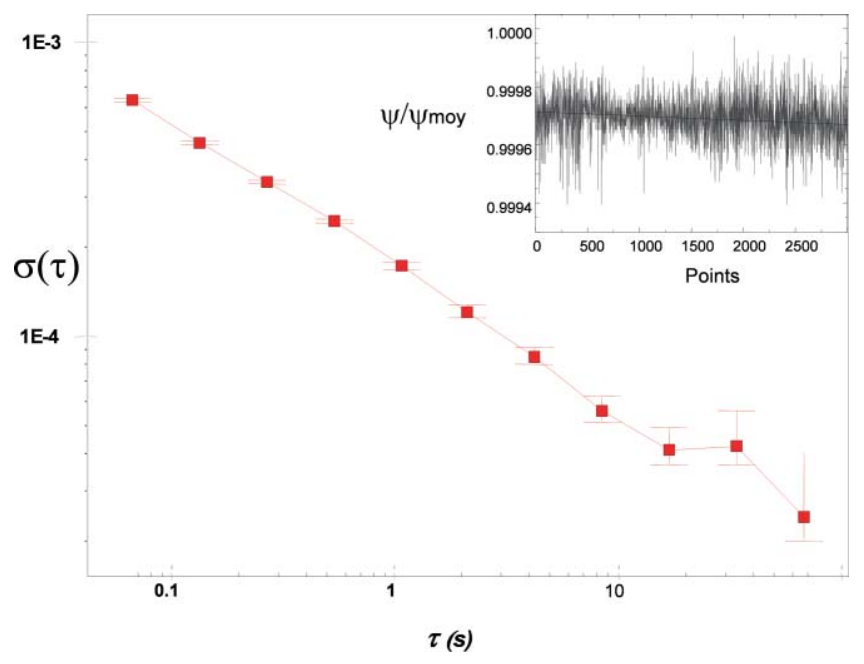

FIG. 4. (Color online) Allan deviation of the ellipsometric parameter $\psi$ at the output of the device. Normalized value of $\psi$ as a function of time (inset).

recorded. The samples are taken with no dead-time between them.

The result is reported in Fig. 4. It represents the Allan standard deviation, ${ }^{23}$ a mathematical operator intended to estimate the instability level of $\psi$ due to noise processes and expressed mathematically as

$$
\sigma_{y}^{2}(\tau)=\frac{1}{2(K-1)} \sum_{i=0}^{K-2}\left(\overline{\psi_{i+1}}(\tau)-\overline{\psi_{i}}(\tau)\right)^{2},
$$

where $\overline{\psi_{i}}$ is the normalized value of each measurement point of the $K$ samples.

We obtain an Allan deviation of $\sigma(\tau)=6.4 \times 10^{-4}$ over an integration time of $0.1 \mathrm{~s}$ and $\sigma(\tau)=2.4 \times 10^{-5}$ for $\tau$ $=10 \mathrm{~s}$. However, one can notice that the Flicker noise limit is not reached in our case which shows that the measurement could be improved by increasing the integration time. We can assume an instability better than $5 \times 10^{-5} \% / \mathrm{s}$ of the polarization state $\psi$ (inset Fig. 4).

\section{B. Experimental measurements of unknown lasers}

For the purpose of validation of our device, we use the polarimetric wavemeter to measure the wavelength of an extended cavity laser diode and we compare our value to the one given by a commercial wavemeter (WA1000 VIS, Burleigh) with an accuracy of $1 \sigma=1 \times 10^{-6}$ and a measurement rate of $4 \mathrm{~Hz}$. Figure 5 represents the experimental setup.

A single frequency helium-neon laser (ML10 Gold, Renishaw) is used as the reference laser. The frequency of the reference laser has been calibrated in regard to a national reference of the Institut National de Métrologie (LNE, France) and it is equal to $\nu_{0}=473.6121176 \times 10^{12} \mathrm{~Hz}$, i.e., $\lambda_{r}=632.991$ $\mathrm{nm}$. The second laser used as the unknown wavelength laser is an extended cavity laser diode (DL100, Toptica). The wavelength of this second laser is simultaneously measured with the help of our polarimetric wavemeter and with a commercial Michelson wavemeter (WA1000, Burleigh). The values given by this wavemeter need a correction due to the 


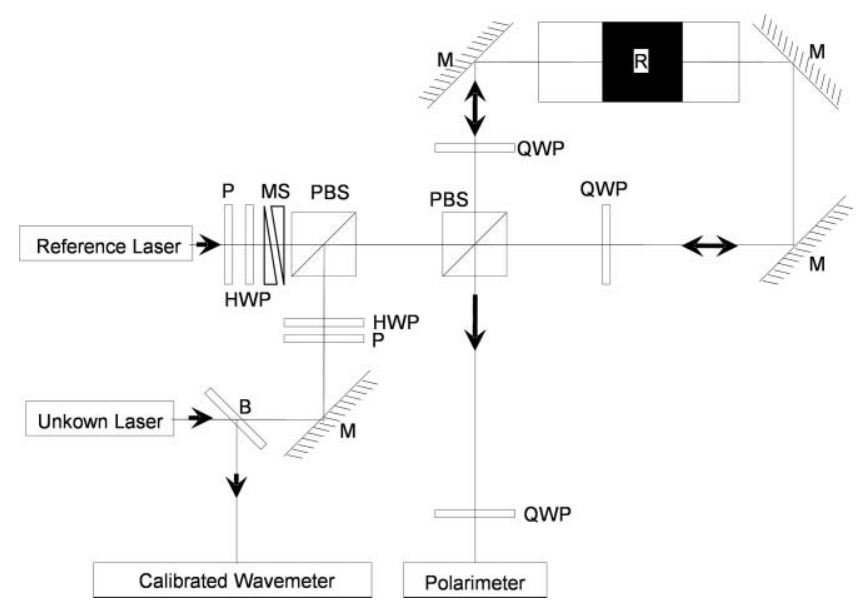

FIG. 5. Example of measurement of the ellipsometric parameter $\psi$ for the reference laser (top) and the unknow laser (bottom).

refractive index of air. For this purpose, a weather station measures the room temperature (PT100 thermistor $-1 \sigma=5 \mathrm{mK}$ ), the room pressure (Digiquartz, Paroscientifics $-1 \sigma=3 \mathrm{~Pa}$ ), and humidity content (MH4, General Eastern $-1 \sigma=1 \%$ ). Using Edlén equations, ${ }^{24}$ the refractive index of air is calculated with an uncertainty of $5 \times 10^{-8}$. A function generator (DS345, Stanford) combined to a voltage amplifier performs the back and forth displacements over $5 \mu \mathrm{m}$ of the retroreflectors mounted onto a piezoelectric actuator (PA840, PI). A mechanical shutter is used to allow one of the lasers at time to pass through the polarimetric wavemeter for a determined

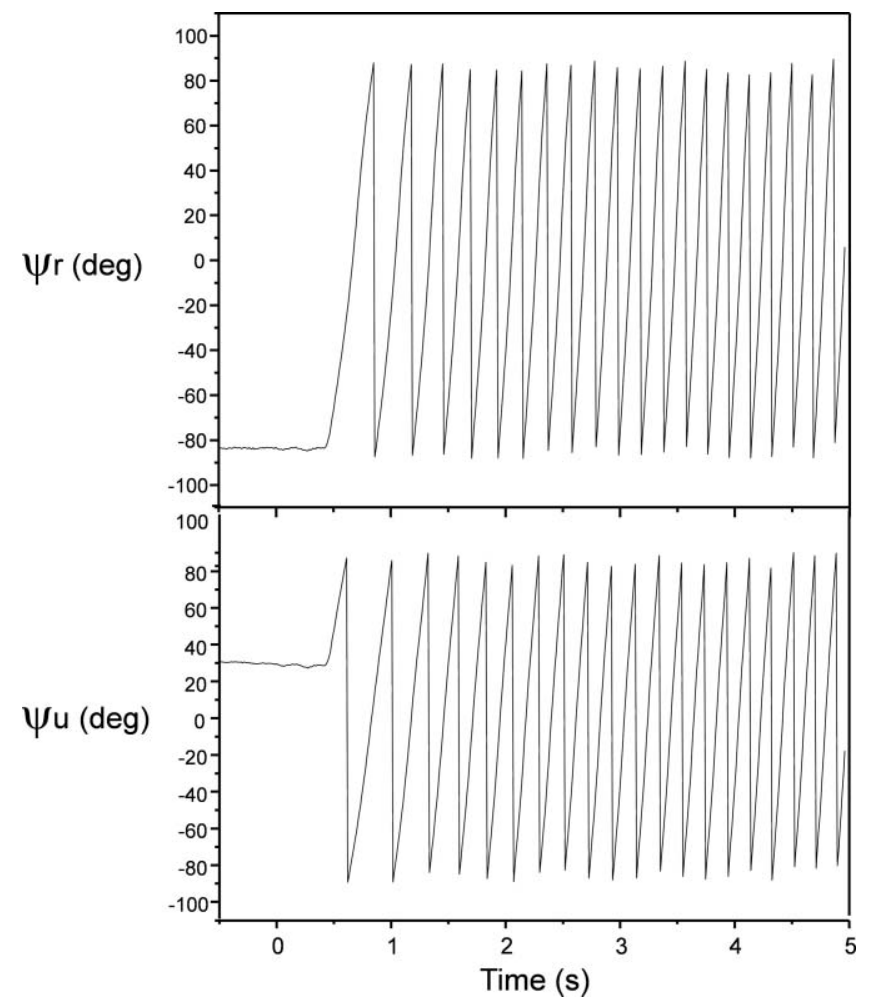

FIG. 6. The experimental setup workbench. B, beamsplitter; HWP, halfwaveplate; M, mirror; MS, mechanical shutter; P, polarizer; PBS, polarizing beamsplitter; QWP, quarter-waveplate; and R, moving retroreflectors.

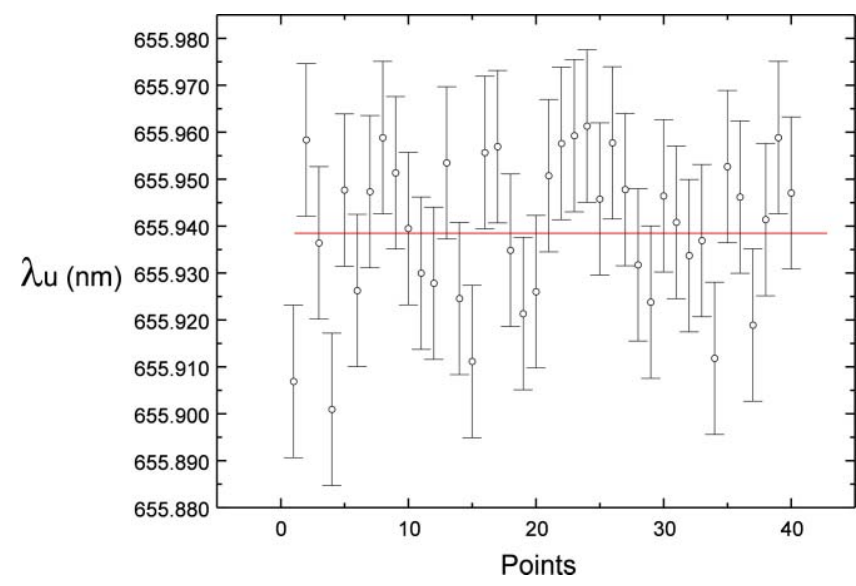

FIG. 7. (Color online) Experimental results of the measurement of the wavelength of a laser beam issue from an extended cavity laser diode. The straight line represents the mean value of the 40 measurement points.

period of time. The data acquisition system of the polarimeter is synchronized during the displacement of the retroreflectors to the mechanical shutter.

Figure 6 represents an example of measurements performed with our wavemeter. One can see the variations of the parameter $\psi$ of both lasers during the displacement.

In this case, the ratio of the wavelengths is $\lambda_{u} / \lambda_{r}$ $=1.036264613$. This leads to a mean value for the unknown wavelength [Eq. (8)] of $\lambda_{u}=655.946 \mathrm{~nm}$. Such measurement has been repeated 40 times. Results have been reported in Fig. 7. After analysis, we obtain a mean value of $\lambda_{u}$ $=655.938 \mathrm{~nm}$ with a standard deviation $1 \sigma=16 \times 10^{-6}$. We observe a discrepancy of $5 \times 10^{-6}$ compared to the value given by the calibrated commercial wavemeter, $\lambda_{u}$ $=655.943(1) \mathrm{nm}$. Same experiment has been repeated with a green line Nd:YAG laser used as the unknown laser. We obtain a value of $532.432(16) \mathrm{nm}$ with the polarimetric wavemeter and 532.422(1) $\mathrm{nm}$ with the calibrated wavemter. Taking into account the error bars, values given by the two wavemeters are in good agreement with the results reported in Fig. 8.

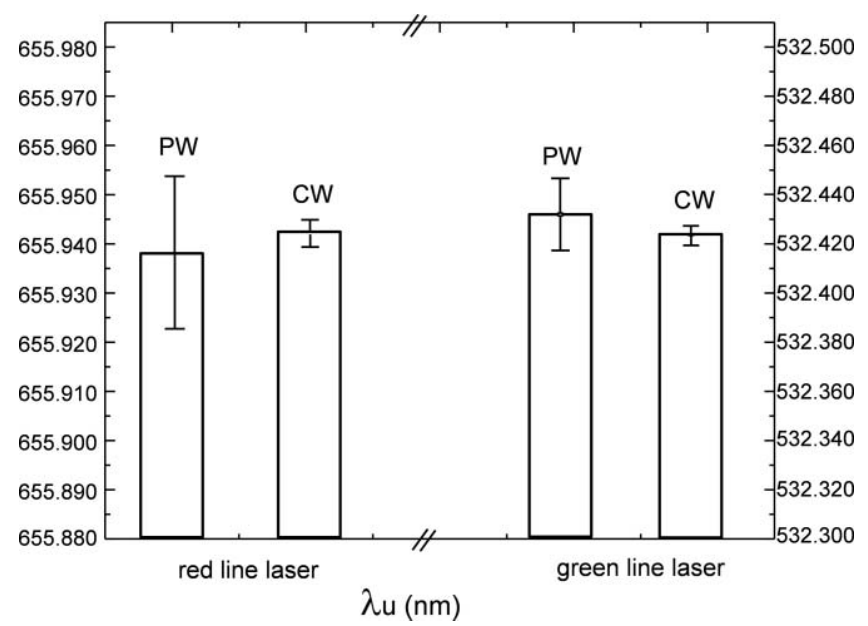

FIG. 8. Experimental measurements of two unknown wavelength lasers with a calibrated wavemeter $(\mathrm{CW})$ and with our polarimetric wavemeter $(\mathrm{PW})$. 


\section{Forthcoming works}

The characteristics of the wavemeter setup, we propose here could be greatly improved with a more advanced design. Free vibrations and thermal isolation could reduce the noise level on the ellipsometric parameters without the need to increase strongly the integration time. Working with a closedloop control displacement and path tracking servo loop control could also permit to reduce this noise level. We expect to achieve a relative accuracy to $10^{-7}$ level with an integration time below $1 \mathrm{~s}$ once these improvements will be appended.

\section{ACKNOWLEDGMENTS}

The authors are grateful to F. Mourgues for his contribution to the design and for the realization of the mechanical parts of the wavemeter.

${ }^{1}$ F. V. Kowalski, R. E. Teets, W. Demtröder, and A. L. Schawlow, J. Opt. Soc. Am. 68, 1611 (1978) .

${ }^{2}$ J. J. Snyder, Laser Focus (Littleton, Mass.) 18, 55 (1982).

${ }^{3}$ J. J. Snyder and T. W. Hansch, in Topics in Applied Physics: Dye Lasers, edited by F. P. Schafer (Springer-Verlag, Berlin, 1990).

${ }^{4}$ A. Banerjee, U. D. Rapol, A. Wasan, and V. Natarajan, Appl. Phys. Lett. 79, 2139 (2001).
${ }^{5}$ L. Yan, B. Chen, W. Yang, R. Wei, and S. Zhao, Rev. Sci. Instrum. 81, 115104 (2010).

${ }^{6}$ J.-P. Monchalin, M. J. Kelly, J. E. Thomas, N. A. Kurnit, A. Szoke, F. Zernike, P. H. Lee, and A. Javan, Appl. Opt. 20, 736 (1981).

${ }^{7}$ P. J. Fox, R. E. Scholten, M. R. Walkiewicz, and R. E. Drullinger, Am. J. Phys. 67, 624 (1999).

${ }^{8}$ M.-L. Junttila, B. Stahlberg, E. Kyro, T. Verijola, and J. Kauppinen, Rev. Sci. Instrum. 58, 1180 (1987).

${ }^{9}$ M. Wakim, S. Topcu, L. Chassagne, J. Nasser, Y. Alayli, and P. Juncar, Opt. Comm. 262, 97 (2006).

${ }^{10}$ J. L. Hall and S. A. Lee, Appl. Phys. Lett. 29, 367 (1976).

${ }^{11}$ R. Castell, W. Demtroder, A. Fisher, R. Kullmer, H. Weickenmeier, and K. Wickert, Appl. Phys. B 38, 1 (1985).

${ }^{12}$ D. F. Gray, K. A. Smith, and F. B. Dunning, Appl. Opt. 25, 1339 (1986).

${ }^{13}$ D. Steers, W. Sibbett, and M. J. Padgett, Appl. Opt. 37, 5777 (1998).

${ }^{14}$ C. Reiser and R. B. Lopert, Appl. Opt. 27, 3656 (1988).

${ }^{15}$ B. Faust and L. Klynning, Appl. Opt. 30, 5254 (1991).

${ }^{16} \mathrm{~S}$. Topçu, L. Chassagne, Y. Alayli, and P. Juncar, Opt. Comm. 247, 133 (2005).

${ }^{17}$ S. Xu, K. Ouedraogo, L. Chassagne, S. Topçu, P. Juncar, and Y. Alayli, Rev. Sci. Instrum. 79, 125104 (2008).

${ }^{18}$ D. W. Berreman, J. Opt. Soc. Am. 62, 502 (1972).

${ }^{19}$ R. C. Jones, J. Opt. Soc. Am. 31, 488 (1941).

${ }^{20}$ R. C. Jones, J. Opt. Soc. Am. 31, 500 (1941).

${ }^{21}$ R. C. Jones, J. Opt. Soc. Am. 32, 486 (1942).

${ }^{22}$ R. M. A. Azzam and N. M. Bashara, in Ellipsometry and Polarized Light, (Elsevier, Amsterdam, 1989).

${ }^{23}$ D. Allan, Proc.-IEEE Ultrason. Symp. 54, 221 (1966).

${ }^{24}$ K. P. Birch and M. J. Downs, Metrologia 30, 155 (1993). 